\title{
Analysis of Security Level Image Steganography Using Secret Key and Gray Codes
}

\author{
Danny Sihombing ${ }^{1}$, Poltak Sihombing ${ }^{1}$ and Rahmat Widia Sembiring ${ }^{1}$ \\ \{dani_aseven@yahoo.com\} \\ ${ }^{1}$ Department of Computer Science, Universitas Sumatera Utara, Medan, Indonesia
}

\begin{abstract}
In Dagar et al (2013) research, message concealment into grayscale image file with Least Significant Bit (LSB) algorithm, where before it is inserted, the message is encrypted with function SN function and converted into binary value from gray code. The result of this research is that resistance to image manipulation is entered into intermediate level where the stego image of insertion result is not resistant to compression and addition of brightness. So in this study suggested to increase data security, resistance to image manipulation and efficiently hide data and time of extraction. In this research we have done Analysis of Security Level of Image Steganography Using Secret Key and Gray Codes with result obtained that on MSE parameter and image size, SNS Function Gray MLSB algorithm has image with MSE and the smallest file size compared to SN Function Gray LSB algorithm has an image with MSE and the largest file size. For process time parameters, it is concluded that each algorithm has almost the same processing time depending on the size of the image and the message to be inserted. SN Function Gray MLSB algorithm based on MSE parameter shows that, process time and image size is better than SN Function Gray LSB algorithm.
\end{abstract}

Keywords: Cryptography, Steganography, Least Significant Bit algorithm, Modified Least Significant Bit.

\section{Introduction}

In Dagar et al (2013) research, message concealment into grayscale image file with Least Significant Bit (LSB) algorithm, where before it is inserted, the message is encrypted with function SN function and converted into binary value from gray code. The inserted image format is the BMP format with grayscale color mode. The result of this research is resistance to image manipulation is entering into middle level, meaning stego image insertion result can not stand against compression, addition of brightness and other image processing. In this study it is suggested to add data security, resilience to image manipulation and efficient in the ability to hide data, data insertion and time of extraction.

In this research, data security will be improved on Dagar et al research by encrypting text data using Secret Key and Gray Codes algorithm and inserting ciphertext into image file (cover image) with MLSB algorithm. The insertion is performed on pixels that are randomized in RGB color 
mode. So if the steganalysis is about to detect the embed, then the embed can not know the location of the pixel and its contents are still in the encrypted state.

\section{Sn Function And Gray Code}

Gray-code or also known as reflected binary code named by Frank Gray. Gray code is a binary numbering system where two adjacent values only have exactly one digit difference. Gray-code was originally used to prevent the false output of an electromechanical signal. Today, Gray-code is used extensively to facilitate error correction in digital communications.

In the hiding data is encrypted using a symmetric key (ks) which is known to both sender and receiver. Data is encrypted using SN function which uses gray code and symmetric key. The main advantage of SN function is that it can encrypt the plain text as well as decrypt the cipher text with small changes. So in this way SN function is very easy to understand and implement (Dagar et al2013).

The SN Function cryptography algorithm is a Gray Code function algorithm in binary form that uses incrementing methods that differ from one digit to the next (Varalakshmi, R. \& Uthariaraj, V. R. 2013). Gray Code is the most popular type of absolute output encoder because its use prevents certain data errors that can occur with Natural Binary during changing circumstances.

For example, in highly capacitive circuits or sluggish system responses, a natural binary state change from condition 0011 to 0100 can cause counters to look like a 0111. This kind of error is not possible with Gray Code, so the data is more reliable. With a gray code, only one bit changes from one position to another. This feature allows the system designer to check the error that if more than one bit changed, then the data can be said not true. The respresentation of decimal numbers, natural binary and Gray Code can be seen as in Table 1 .

\begin{tabular}{|c|c|c|}
\hline Decimal & Natural Binary & Gray Code \\
\hline 0 & 0000 & 0000 \\
\hline 1 & 0001 & 0001 \\
\hline 2 & 0010 & 0011 \\
\hline 3 & 0011 & 0010 \\
\hline 4 & 0100 & 0110 \\
\hline 5 & 0101 & 0111 \\
\hline 7 & 0110 & 0101 \\
\hline 8 & 0111 & 0100 \\
\hline 9 & 1000 & 1100 \\
\hline 10 & 1001 & 1101 \\
\hline
\end{tabular}

Table 1. Decimal Representation, Natural Binary and Gray Code

Conversion from Gray Code to Binary Code

Let Gray Code be g3 g2 g1 g0. Then the respective Binary Code can be obtained as follows:

Gray code $\quad: g_{3} \quad g_{2} \quad g_{1} g_{0}$ 


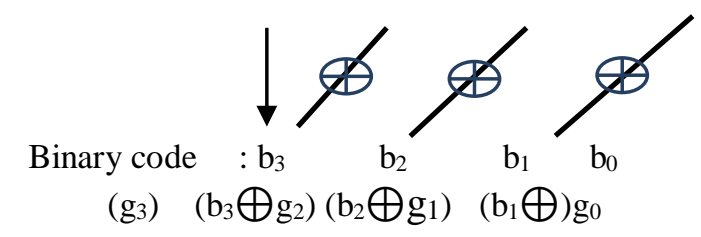

Let Binary code be b3 b2 b1 b0. Then the respective Gray Code can be obtained is as follows

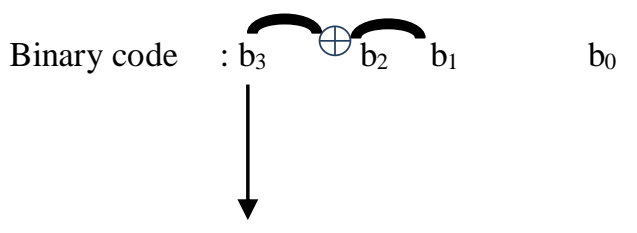

Gray code $\quad: g_{3} g_{2} \quad g_{1} \quad g_{0}$
$\left(\mathrm{b}_{3}\right)(\mathrm{b} 3 \bigoplus 2)(\mathrm{b} 2$
(17) (b1 b甲)

\section{Modifiedleast Significant Bit (MLSB)}

Modified Least Significant Bit (MLSB) or modification of the LSB algorithm is used to encode an identity into a file. MLSB uses the manipulation of several levels of insertion bits before encoding the message (Zaher, 2011).Modify messages with MLSB algorithm where message bits that should have 1 character have an 8 bit ASCII code will be modified to 5 bits. In this algorithm characters and numbers are represented in 5 bits which will then be inserted into the original file by LSB technique. The insertion is done by processes:

1. The process of altering insert data with ASCII code. For example the message "STEGO with 05 bits" which if converted to binary requires memory of $18 \times 8$ bits $=144$ bits. In the MLSB algorithm the above message is converted to ASCII (hex) to: 53h, 54h, 45h, 47h, 4fh, 20h, 77h, $69 \mathrm{~h}, 74 \mathrm{~h}, 68 \mathrm{~h}, 20 \mathrm{~h}, 30 \mathrm{~h}, 35 \mathrm{~h}, 20 \mathrm{~h}, 62 \mathrm{~h}, 69 \mathrm{~h}, 74 \mathrm{~h}, 73 \mathrm{~h}$. Then done normalization with table Control Symbol like Table 2.

Table 2. Control Symbol

\begin{tabular}{|l|l|}
\hline Hex Representation & Operation \\
\hline $1 \mathrm{Bh}$ & Define Small Letter \\
\hline $1 \mathrm{Ch}$ & Define Capital Letter \\
\hline $1 \mathrm{Dh}$ & Define Space \\
\hline $1 \mathrm{Eh}$ & Define Number \\
\hline $1 \mathrm{Fh}$ & Define end of text \\
\hline
\end{tabular}


2. Read the insertion data (ASCII) until the space mark (20h) is 53, 54, 45, 47, 4f.

3. All values are reduced by the lowest value of 40 to $53-40=13,54-40=14,45-40=05,47-40$ $=07,4 \mathrm{f}-40=\mathrm{f}$.

4. The first group insertion data is $1 \mathrm{ch}, 13 \mathrm{~h}, 14 \mathrm{~h}, 05 \mathrm{~h}, 07 \mathrm{~h}, 0 \mathrm{f}$ where $1 \mathrm{ch}$ is the Control Symbol for uppercase (capital).

5. The second group insertion data is $77 \mathrm{~h}, 69 \mathrm{~h}, 74 \mathrm{~h}, 68 \mathrm{~h}$ reduced by the lowest value (60) to 77 $60=17,69-60=09,74-60=14,68-60=08$.

6. The second group data is combined with the first group and assigned a Control Symbol 1dh (space) and 1bh (lowercase) to $1 \mathrm{dh}, 1 \mathrm{bh}, 17 \mathrm{~h}, 09 \mathrm{~h}, 14 \mathrm{~h}, 08 \mathrm{~h}$.

7. The third group data are: $30 \mathrm{~h}, 35 \mathrm{~h}$ minus the lowest value being: $30-30=0,35-30=05$.

8. The data is combined with the previous group plus Control Symbol 1dh (space), 1eh (number) to $1 \mathrm{dh}, 1 \mathrm{eh}, 00 \mathrm{~h}, 05 \mathrm{~h}$.

9. The data of the fourth group are: $62 \mathrm{~h}, 69 \mathrm{~h}, 74 \mathrm{~h}, 73 \mathrm{~h}$ reduced by the lowest value to: $62-60=$ $02,69-60=09,74-60=14,73-60=13$.

10. The data is combined with the previous group plus Control Symbol 1bh (lowercase), into 1dh, 1bh, 02h, 09h, 14h, 13h and end of data (1fh).

So the message becomes:

$1 \mathrm{ch}, 13 \mathrm{~h}, 14 \mathrm{~h}, 05 \mathrm{~h}, 07 \mathrm{~h}, 0 \mathrm{hh}, 1 \mathrm{hh}, 08 \mathrm{~h}, 1 \mathrm{hh}, 1 \mathrm{~h}, 00 \mathrm{~h}, 05 \mathrm{~h}, 1 \mathrm{bh}, 02 \mathrm{~h}, 09 \mathrm{~h}, 14 \mathrm{~h}, 13 \mathrm{~h}, 1 \mathrm{fh}$. The above message requires $22 \times 5$ bits $=110$ bits and converted to binary to:

11100, 10011, 10100, 00101, 00111, 01111, 11101, 11011, 10111, 01001, 10100, 01000, 11101, 11110, 00000, 00101, 11011, 00010, 01001, 10100, 10011, 11111.

\section{Method Of Analysis}

In this research, the analysis adds data security level to image file with cryptography technique and steganography on Dagar et al. Secured data in the form of secret text that is inserted into digital image files that are in BMP, JPG or PNG format with Modified Least Significant Bit (Modified-LSB) algorithm. Before being inserted, secret text is encrypted with SN function algorithm using Gray Code. As a comparison in this study, data security is done by SN encryption algorithm with Gray Code and insertion with Least Sifnificant Bit (LSB) algorithm. To measure the reliability of the insertion of each algorithm, measurements of size, dimensions, Mean Squared Error (MSE) and Peak Signal to Noise Ratio (PSNR) values of the stego image were measured. And to measure the reliability of data acquisition embed, then calculate the value of Data Recovery Rate (DRR) as the value of extraction.

The Flowchart Research consists of two parts namely the flowchart insertion and extraction. Flowchart Insertion is a process flow for inserting text files into image media (cover image) to generate stego image, while flowchart Extraction is a process flow to extract insert data from stego image. Flowchart insertion can be seen as in Figure 1.

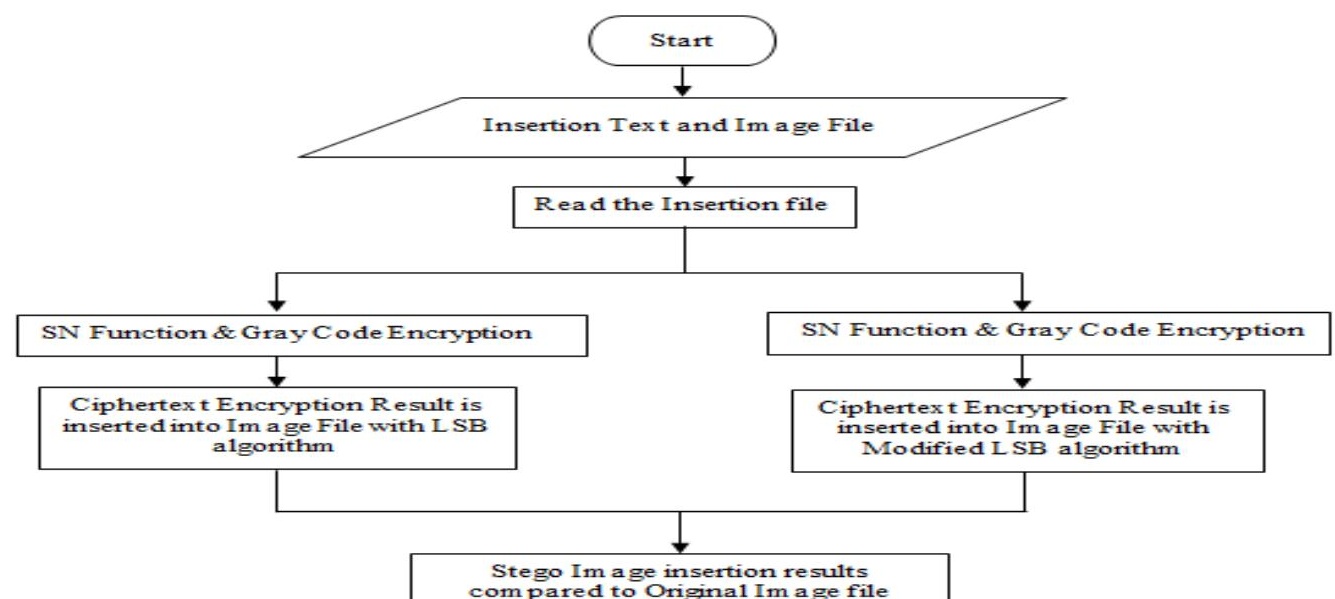


Figure 1. Flowchart Insertion

Information:

In the flowchart above there are two paths of encryption and insertion process are:

1. Encryption algorithm SN function Gray Code LSB

In this algorithm is inserted data input txt format, then do the reading file to get data and then done by encryption to insert with LSB algorithm to get ciphertext file. The ciphertext file obtained is inserted into the image file with MLSB algorithm and then calculated the value of MSE and PSNR the stego image file obtained.

\section{Encryption algorithm SN function and Gray Code Modified-LSB}

In this algorithm input data input inserted txt format, then do the reading file to obtain data and then done by encryption to insert with Modified-LSB algorithm to get ciphertext file. The ciphertext file obtained is inserted into the image file with the Modified-LSB algorithm and then the MSE and PSNR values of the stego image file are obtained. Flowchart Extraction of insert data from the stego image file can be seen as in Figure 2.

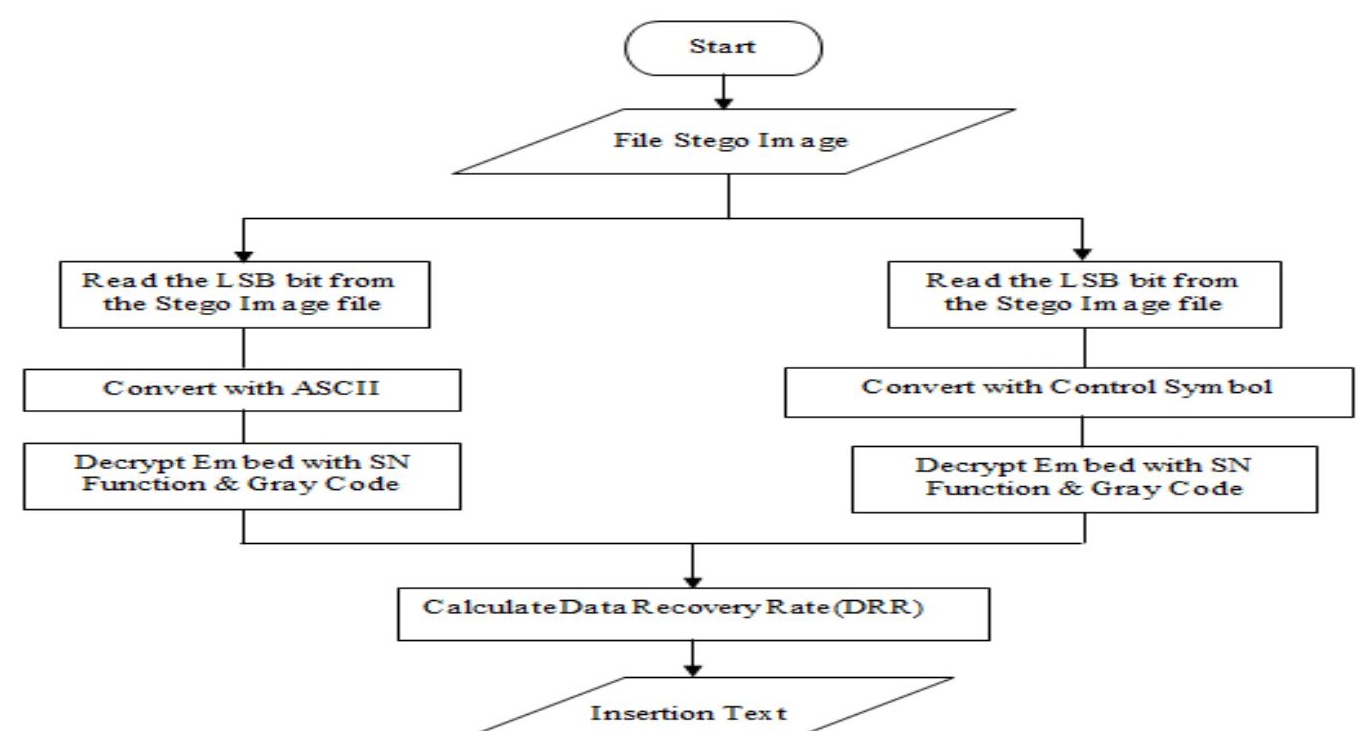


Figure 2. Flowchart Extraction

Information:

In the flowchart above the input stego image file that has been inserted, then read LSB bits and and converted into ASCII and Control Symbol. After conversion, the embedded decryption is done with SN Function and Modified-LSB algorithm to obtain the data of encrypted bits, then done decryption process to the insert file to get the plaintext and then calculate the value of Data Recovery Rate (DRR) as the extraction value.

\section{Insertion of Sn Function and Gray Code Lgorithms}

The insertion algorithm with SN Function and Gray Code is the insertion of the text of a secret message into a digital image file in BMP, JPG or PNG format with the LSB algorithm. Before being inserted, the first text message is encrypted using Gray Code and SN Function. The insertion steps with SN Function and Gray Code are as follows:

1. For example, the pixel value of the cover image is $8 \times 5$ pixels as shown in Table 3 .

Table 3. Cover Image Matrix

\begin{tabular}{|l|l|l|l|l|l|l|l|}
\hline 50 & 10 & 60 & 50 & 58 & 40 & 44 & 12 \\
\hline 10 & 52 & 40 & 20 & 34 & 74 & 30 & 87 \\
\hline 12 & 24 & 45 & 25 & 60 & 28 & 20 & 77 \\
\hline
\end{tabular}




\begin{tabular}{|l|l|l|l|l|l|l|l|}
\hline 10 & 60 & 40 & 30 & 54 & 32 & 24 & 85 \\
\hline 12 & 55 & 22 & 24 & 33 & 45 & 37 & 110 \\
\hline
\end{tabular}

2. Convert insert text into binary value, for example the text is "DANI" with binary is:

$\mathrm{D}=01000100$

$\mathrm{A}=01000001$

$\mathrm{N}=01001110$

$\mathrm{I}=01001001$

3. Formation of Ciphertext Gray Code with SN Function Encryption

Plain Text D is 01000100

Key 10011001

Encryption: (Plain Text) XNOR (Key) (same equals = 1 else 0) 01000100

XNOR 10011001

00100010

Gray code for 00100010 is 00110011

Cipher text $\mathrm{D}=00110011$

So cipherteks "DANI" is 00110011010000010011110000111000 . Binary messages are inserted into an Image cover file as in Table 4.

Table 4. Cover Image Matrix

\begin{tabular}{|l|l|l|l|l|l|l|l|}
\hline 50 & 10 & 60 & 50 & 58 & 40 & 44 & 12 \\
\hline 10 & 52 & 40 & 20 & 34 & 74 & 30 & 87 \\
\hline 12 & 24 & 45 & 25 & 60 & 28 & 20 & 77 \\
\hline 10 & 60 & 40 & 30 & 54 & 32 & 24 & 85 \\
\hline 12 & 55 & 22 & 24 & 33 & 45 & 37 & 110 \\
\hline
\end{tabular}

4. Convert The image cover pixel value in Table 4 above is converted into binary into Table 5 .

Table 5. Binary Image Cover Image Matrix

\begin{tabular}{|l|l|l|l|l|l|l|l|}
\hline 00110010 & 00001010 & 00111100 & 00110010 & 00111010 & 00101000 & 00101100 & 00001100 \\
\hline 00001010 & 00110100 & 00101000 & 00010100 & 00100010 & 01001010 & 00011110 & 01010111 \\
\hline 00001100 & 00011000 & 00100101 & 00011001 & 00111100 & 00011100 & 00010100 & 01001101 \\
\hline 00001010 & 00111100 & 00101000 & 00011110 & 00110110 & 00100000 & 00011000 & 01010101 \\
\hline 00001100 & 00110111 & 00010110 & 00011000 & 00100001 & 00101101 & 00100101 & 00110010 \\
\hline
\end{tabular}


5. The insertion is done by the LSB method on each byte on the rear binary value (LSB) as in Table 6.

Table 6. Binary Image Matrix Stego Image

\begin{tabular}{|l|l|l|l|l|l|l|l|}
\hline 00110010 & 00001010 & 00111101 & 00110011 & 00111010 & 00101000 & 00101101 & 00001100 \\
\hline 00001010 & 00110100 & 00101001 & 00010100 & 00100011 & 01001011 & 00011111 & 01010111 \\
\hline 00001100 & 00011000 & 00100101 & 00011001 & 00111101 & 00011100 & 00010100 & 01001100 \\
\hline 00001010 & 00111100 & 00101001 & 00011111 & 00110110 & 00100001 & 00011001 & 01010101 \\
\hline 00001100 & 00110110 & 00010111 & 00011001 & 00100001 & 00101100 & 00100101 & 00110011 \\
\hline
\end{tabular}

After insertion, the representation of the stego image pixel value becomes as in Table 7.

Table 7. Grayscale Image Stego Image Matrix

\begin{tabular}{|l|l|l|l|l|l|l|l|}
\hline 50 & 10 & 61 & 51 & 58 & 40 & 45 & 13 \\
\hline 10 & 52 & 41 & 20 & 35 & 75 & 31 & 87 \\
\hline 12 & 24 & 45 & 25 & 61 & 28 & 20 & 76 \\
\hline 10 & 60 & 41 & 31 & 54 & 33 & 25 & 85 \\
\hline 12 & 54 & 23 & 25 & 33 & 44 & 37 & 111 \\
\hline
\end{tabular}

\section{Findings And Discussion}

The analysis by the author of the security level analysis of Image Steganography using Secret Key \& Gray Codes is to increase the level of data security into image files with cryptographic and steganographic techniques as in the research of Dagar, Kumar and Bagoriya. Secured data in the form of secret text that is inserted into digital image files that are in BMP, JPG or PNG format with the Modified Least Significant Bit (MLSB) algorithm where before being inserted, secret text is encrypted with Secret Key \& Gray Codes algorithm.For comparison, data is inserted into digital image files in BMP, JPG or PNG format with Least Significant Bit (LSB) algorithm and prior to being inserted, in secret text encryption with SN function using Gray Code.

The test is performed using five different sized digital images where each image will be inserted with five different text watermarks. The parameters to be obtained are the MSE, the length of the process and the result file size. From the test results obtained as shown in Table 8. and Table 9.

Table 8. Results of Steganographic Insertion of GrayKey SN Function LSB 


\begin{tabular}{|c|c|c|c|c|c|c|c|}
\hline \multirow[t]{2}{*}{ No } & \multirow[t]{2}{*}{ Cover Img } & \multirow{2}{*}{$\begin{array}{c}\text { Size } \\
\text { (byt) }\end{array}$} & \multirow[t]{2}{*}{ Embed File } & \multirow{2}{*}{$\begin{array}{l}\text { Num } \\
\text { Char }\end{array}$} & \multicolumn{3}{|c|}{ Parameter } \\
\hline & & & & & MSE & PSNR & $\mathrm{t}(\mathrm{S})$ \\
\hline \multirow[t]{5}{*}{1} & Pic01.jpg & 110.020 & Pesan1.txt & 29 & 5.63 & 40.62 & 1.30 \\
\hline & Pic01.jpg & 110.020 & Pesan2.txt & 73 & 15.02 & 36.36 & $1: 28$ \\
\hline & Pic01.jpg & 110.020 & Pesan3.txt & 158 & 29.86 & 33.37 & $1: 32$ \\
\hline & Pic01.jpg & 110.020 & Pesan4.txt & 185 & 34.94 & 32.69 & $2: 02$ \\
\hline & Pic01.jpg & 110.020 & Pesan5.txt & 228 & 47.32 & 31.37 & $2: 15$ \\
\hline \multirow[t]{5}{*}{2} & Pic02.jpg & 148.525 & Pesan1.txt & 29 & 0.33 & 52.85 & $2: 46$ \\
\hline & Pic02.jpg & 148.525 & Pesan2.txt & 73 & 1.16 & 47.47 & $2: 43$ \\
\hline & Pic02.jpg & 148.525 & Pesan 3.txt & 158 & 1.93 & 45.25 & $2: 46$ \\
\hline & Pic02.jpg & 148.525 & Pesan4.txt & 185 & 2.24 & 44.62 & $2: 46$ \\
\hline & Pic02.jpg & 148.525 & Pesan5.txt & 228 & 4.47 & 41.62 & $2: 46$ \\
\hline \multirow[t]{5}{*}{3} & Pic03.jpg & 194.710 & Pesan1.txt & 29 & 0.029 & 63.48 & $1: 28$ \\
\hline & Pic03.jpg & 194.710 & Pesan2.txt & 73 & 1.41 & 46.63 & $1: 34$ \\
\hline & Pic03.jpg & 194.710 & Pesan3.txt & 158 & 1.82 & 45.52 & $1: 34$ \\
\hline & Pic03.jpg & 194.710 & Pesan4.txt & 185 & 2.33 & 44.45 & $1: 35$ \\
\hline & Pic03.jpg & 194.710 & Pesan5.txt & 228 & 5.28 & 40.90 & $1: 36$ \\
\hline \multirow[t]{5}{*}{4.} & Pic04.jpg & 138.358 & Pesan1.txt & 29 & 2.16 & 44.78 & $3: 55$ \\
\hline & Pic04.jpg & 138.358 & Pesan2.txt & 73 & 6.86 & 39.76 & $3: 54$ \\
\hline & Pic04.jpg & 138.358 & Pesan3.txt & 158 & 11.46 & 11.46 & 37.53 \\
\hline & Pic04.jpg & 138.358 & Pesan4.txt & 185 & 13.41 & 13.41 & 36.85 \\
\hline & Pic04.jpg & 138.358 & Pesan5.txt & 228 & 22.64 & 22.64 & 34.58 \\
\hline \multirow[t]{5}{*}{5} & Pic05.jpg & 223.932 & Pesan1.txt & 29 & 2.30 & 2.30 & 44.49 \\
\hline & Pic05.jpg & 223.932 & Pesan2.txt & 73 & 7.68 & 7.68 & 39.27 \\
\hline & Pic05.jpg & 223.932 & Pesan3.txt & 158 & 12.26 & 12.26 & 37.24 \\
\hline & Pic05.jpg & 223.932 & Pesan4.txt & 185 & 14.34 & 14.34 & 36.56 \\
\hline & Pic05.jpg & 223.932 & Pesan5.txt & 228 & 25.55 & 25.55 & 34.05 \\
\hline
\end{tabular}

Table 9. Insertion Results of GrayKey SN Function Modified-LSB Steganography 


\begin{tabular}{|l|l|l|l|c|c|c|r|} 
No & Cover Img & Size & Embed File & Num. Char & \multicolumn{3}{|c|}{ Paramter } \\
\cline { 5 - 8 } & & & & MSE & PSNR & t(S) \\
\hline 1 & Pic01.jpg & 110.020 & Pesan1.txt & 29 & 0.000035 & 92.65 & $1: 2$ \\
\hline & Pic01.jpg & 110.020 & Pesan2.txt & 73 & 0.000084 & 88.84 & $1: 2$ \\
\hline & Pic01.jpg & 110.020 & Pesan3.txt & 158 & 0.000165 & 85.93 & 1.2 \\
\hline & Pic01.jpg & 110.020 & Pesan4.txt & 185 & 0.000197 & 85.17 & $1: 2$ \\
\hline & Pic01.jpg & 110.020 & Pesan5.txt & 228 & 0.000281 & 83.63 & $1: 2$ \\
\hline 2 & Pic02.jpg & 148.525 & Pesan1.txt & 29 & 0.000024 & 94.31 & $2: 4$ \\
\hline & Pic02.jpg & 148.525 & Pesan2.txt & 73 & 0.000052 & 90.92 & $2: 4$ \\
\hline & Pic02.jpg & 148.525 & Pesan3.txt & 158 & 0.000097 & 88.22 & $2: 4$ \\
\hline & Pic02.jpg & 148.525 & Pesan4.txt & 185 & 0.000098 & 88.19 & $2: 4$ \\
\hline & Pic02.jpg & 148.525 & Pesan5.txt & 228 & 0.000134 & 86.84 & $2: 4$ \\
\hline 3 & Pic03.jpg & 194.710 & Pesan1.txt & 29 & 0.000034 & 92.72 & $1: 3$ \\
\hline & Pic03.jpg & 194.710 & Pesan2.txt & 73 & 0.000089 & 88.60 & $1: 3$ \\
\hline & Pic03.jpg & 194.710 & Pesan3.txt & 158 & 0.000155 & 86.21 & $1: 3$ \\
\hline & Pic03.jpg & 194.710 & Pesan4.txt & 185 & 0.000182 & 85.51 & $1: 3$ \\
\hline & Pic03.jpg & 194.710 & Pesan5.txt & 228 & 0.000216 & 84.77 & $1: 3$ \\
\hline 4 & Pic04.jpg & 138.358 & Pesan1.txt & 29 & 0.000016 & 95.87 & $3: 4$ \\
\hline & Pic04.jpg & 138.358 & Pesan2.txt & 73 & 0.000015 & 91.94 & $3: 4$ \\
\hline & Pic04.jpg & 138.358 & Pesan3.txt & 158 & 0.000075 & 89.58 & $3: 4$ \\
\hline & Pic04.jpg & 138.358 & Pesan4.txt & 185 & 0.000098 & 88.17 & $3: 4$ \\
\hline & Pic04.jpg & 138.358 & Pesan5.txt & 228 & 0.000120 & 87.30 & $3: 4$ \\
\hline 5 & Pic05.jpg & 223.932 & Pesan1.txt & 29 & 0.000014 & 96.65 & $3: 2$ \\
\hline & Pic05.jpg & 223.932 & Pesan2.txt & 73 & 0.000039 & 92.17 & $3: 3$ \\
\hline & Pic05.jpg & 223.932 & Pesan3.txt & 158 & 0.000101 & 88.05 & $3: 3$ \\
\hline & Pic05.jpg & 223.932 & Pesan4.txt & 185 & 0.000084 & 86.66 & $3: 3$ \\
\hline & Pic05.jpg & 223.932 & Pesan5.txt & 228 & 0.000111 & 87.66 & $3: 3$ \\
\hline
\end{tabular}

\section{Conclusion}

Based on comparison of SN Function Gray LSB and SN Function Gray MLSB algorithm on three comparison parameters: MSE image, process time and image size obtained that on MSE parameter and image size, SN Function Gray MLSB algorithm has image with MSE and the smallest file size compared to the SN Function Gray LSB algorithm that has the image with MSE and the largest file size. For process time parameters, it is concluded that each algorithm has the 
same processing time depends on the size of the image and the message to be inserted. SN Function Gray MLSB algorithm data show that based on MSE image parameter, process time and image size is above SN Function Gray LSB algorithm.

\section{References}

[1] Gonzalez, R.C. \& Woods, R.E. 2003. Digital Image Processing. third edition, USA: Addison-Wesley Publishing Co, University of Tennessee.

[2] Sridevi, R. Damodaram, A. \& Narasimham. 2005. Efficient Method Of Audio Steganography by Modified LSB Algorithm And Strong Encryption Key With Enhanced Security. Journal of Theoretical and Applied Information Technology, Vol 37, No 22. Jntuceh, Hyderabad India. Maret 2005 : 768-771.

[3] Zaher, M.A. 2011. Modified Least Significant Bit (MLSB). Jurnal Computer and Information Science Vol. 4, No. 1, Januari 2011. www.ccsenet.org/cis. Diakses tanggal 15 Maret 2012 : 60-67.

[4] Dagar, S., Kumar, V. \& Bagoriya Y. 2013. Image Steganography using Secret Key \& Gray Codes. (IJITEE) ISSN: 2278-3075, Volume-2, Issue-5, April 2013.

[5] Shreya, M.S. \& Khumar, S. 2013. Separable Reversible Data Hiding In Encrypted Image Using Modified Least Significant Bit and Virtual Embedding. International Journal of Science and Research. Volume 4, Issue 4, April 2015 : 3099-3106.

[6] Hammood, D.A. 2013. Breaking A Playfair Cipher Using Memetic Algorithm. Journal of Engineering and Development, Vol. 17, No.5, November 2013. College of Elec. \& Electronic Techniques Foundation of Technical Education.

[7] Prasetiyo, B. 2013. Kombinasi Steganografi Bit Matchingdan Kriptografi DES untuk Pengamanan Data. Tesis. Universitas Diponegoro : Semarang.

[8] Sentilkumar, M. \& Mathivanan, V. 2016. Analysis of Data Compression Tecniques using Huffman Coding and Arithmetic Coding. International Journal of Advanced Research in Computer Science and Software Engineering. Vol. 6, Issue 5, May 2016 : 930-936.

[9] Singh, S. \& Siddiqui, T.J. 2012. A Security Enhanced Robust Steganography Algorithm for Data Hiding. International Journal of Computer ScienceIssues. Volume 9, Issue 3, No. 1, May 2012 : 131 139.

[10] Husain, M.P. \& Rafat, K.F. 2016. Enhanced Audio LSB Steganography for Secure Communication. International Journal of Advanced Computer Science and Aplications, Vol. 7, No. $1: 340-347$.

[11] Kaul, N. , Bajaj, N. 2013. Audio in Image Steganography based on Wavelet Transform. International Journal of Computer Application. Vol. 79- No. 03, October 2013: 7-10.

[12] Varalakshmi, R. \& Uthariaraj, V. R. 2013. Error Correction for a Secure Multicast Group Key Management using Gray Code. International Journal of Computer Applications (0975 - 8887) Volume 66- No.1, March 2013. Ramanujan Computing Centre, Anna University Chennai, Tamil Nadu, India. 